\title{
Adjuvant urokinase: percutaneous drainage of post lower segment cesarean section intra-abdominal abscess
}

\author{
Shweta Avinash Khade*, Balaji Jadhav, Preeti Meena
}

Department of Obstetrics and Gynecology, BYL TNMC Nair Hospital, Mumbai Central, Maharashtra, India

Received: 27 February 2019

Accepted: 02 April 2019

\section{*Correspondence:}

Dr. Shweta Avinash Khade,

E-mail: drshwetam2009@gmail.com

Copyright: ( ) the author(s), publisher and licensee Medip Academy. This is an open-access article distributed under the terms of the Creative Commons Attribution Non-Commercial License, which permits unrestricted non-commercial use, distribution, and reproduction in any medium, provided the original work is properly cited.

\begin{abstract}
The mortality in abdominal abscess is high, however the outcome has improved due to advances in image guided percutaneous interventional techniques. The main indications for the catheter drainage include treatment or palliation of sepsis associated with an infected fluid collection, and alleviation of the symptoms that may be caused by fluid collections by virtue of their size, and site. The single abscesses may be drained with ultrasound guidance only, whereas the multiple abscesses usually require computed tomography (CT) guidance and placement of multiple catheters. Percutaneous drainage provides an effective and safe alternative to more invasive surgical drainage but the success rate is lower for abscesses that have septa and are multilocular. Several clinical and in vitro studies suggest urokinase may be useful in such cases. To the knowledge, however, there has been no case of post LSCS intraabdominal abscess in which intracavitary urokinase was administered. Therefore, we report a case of post LSCS multiseptated intra-abdominal abscess occurring in a 21-year-female. Conventional percutaneous tube drainage failed, but the use of transcatheter intracavitary urokinase was successful. Our results showed no significant change in hematologic studies and no bleeding complications. Intracavitary urokinase can be given safely during percutaneous drainage of an abscess, with no associated bleeding complications or changes in coagulation parameters.
\end{abstract}

Keywords: Intra-abdominal abscess, Percutaneous drainage, Urokinase

\section{INTRODUCTION}

Percutaneous drainage of an abscess is accepted as an effective alternative to surgical drainage, but the success rate for the drainage of septate or multilocular abscesses is lower than for simple abscesses. ${ }^{1}$ Several clinical and in vitro studies suggest urokinase may be useful in such cases. $^{2}$

Intrapleural fibrinolytic therapy to accelerate drainage of loculated hemothorax and empyema has been reported. ${ }^{3,4}$ Volgelzang et al, described the successful use of transcatheter intracavitary fibrolysis in the treatment of infected intra-abdominal postsurgical hematomas in two patients. ${ }^{5}$ To our knowledge, there is no literature of its use in postpartum intra-abdominal abscess cases. We describe here the successful use of transcatheter intracavitary urokinase in the treatment of a post LSCS multiloculated pyogenic abscess in our patient.

\section{CASE REPORT}

A 21-year-old, P1L1IUFD1 day 17 post LSCS was admitted in emergency for the evaluation of pain in abdomen and fever for 15 days

Patient had undergone emergency LSCS for twin gestation with imminent eclampsia in a private Hospital at Secunderabad. Post LSCS Patient had complaint of fever, burning micturition and loose stool $\mathrm{s}$ with gradual 
abdominal distension. Patient received antibiotics but no relief obtained so referred to our hospital.

On admission patient was febrile, Pulse rate $=120 / \mathrm{min}$, $\mathrm{BP}=110 / 80 \mathrm{mmhg}$. Systemic examination was normal.

Abdominal examination revealed distended abdomen with oedematous and indurated suture site. Pus was draining out from right angle of pfannenstiel incision.

On per vagina examination uterus was bulky, bilateral forniceal fullness and tenderness was present. No per vaginal discharge seen.

On blood investigation hb-9.5 gm\% WBC count was raised i.e. 22,000 with neutrophilic lymphocytosis.

Abdominal pelvis ultrasound revealed a localised heterogenous predominantly hypoechoic collection of $14 \times 12 \times 15 \mathrm{~cm}$ (approx.1400cc) in the peritoneal cavity reaching upto epigastrium with thick septations within, and heterogenous collection $5 * 5 \mathrm{~cm}$ in anterior abdominal wall.

Abdominopelvic computed tomography $(\mathrm{P}+\mathrm{C})$ shows loculated sub peritoneal collection measuring $18 \times 8.6 \times 16.5 \mathrm{~cm}$ with air pockets in it and extending from pelvis upto umbilicus, displacing bowel loops posteriorly. $21 * 90 \mathrm{~mm}$ heterogenous collection in anterior abdominal wall at operative site.

The preliminary diagnosis was pyogenic localized intraabdominal abscess was made.

Systemic antibiotics therapy was begun and ultrasoundguided percutaneous draining catheter was placed in the abscess pocket. To keep the catheter lumen clear, 10-15 cc salines was injected every 8 hours. 20cc purulent material was aspirated through the tube and sent for gram staining, cytologic examination.

Culture sensitivity reveals plenty of pus cells. Suggestive of pyogenic abscess. On pus culture Acinetobacter Organism isolated, sensitive to colistin, an antibiotic was stepped up.

On follow up ultrasound After 3 days size of collection reduced to $400 \mathrm{cc}$.

Thereafter up to 1 week the size of collection remain constant and patient was still symptomatic.

Authors have tried to evacuate purulent material from the cavity by changing percutaneous drain site but only $10 \mathrm{cc}$ pus was removed.

Authors believed that this failure was due to multiloculation and viscous nature of the abscess fluid. To decrease the viscosity of purulent material and break septations decision of use of intracavitary urokinase (local fibrinolytic) was taken.

The abscess cavity is flushed through 12 fr catheter with urokinase 1,20,000 units dissolved in $120 \mathrm{ml}$ normal saline. Once flushing was finished the catheter was closed for $4 \mathrm{hr}$ and then reopened for $2 \mathrm{hr}$ period for gravity drainage. This procedure was repeated for three consecutive days. Saline irrigation was continued for six days. Patient tolerated the drainage procedures and the instillation of urokinase well. No significant complications, including any form of hemorrhage, occurred.



Figure 1: A 21-year-old female with a multiseptated intraabdominal abscess. A CT scan shows a large loculated mass with multiple septations in.

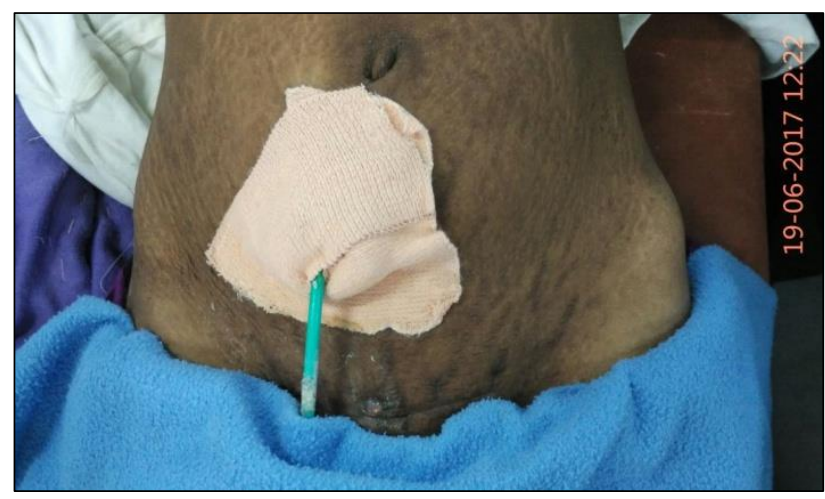

Figure 2: Repeat scan after insertion of catheter shows persistent fluid with several air bubbles. Drainage catheter (arrow) is well demonstrated in the center of abscess cavity on CT after drainage.

At follow up clinical status the daily quantity of purulent material drained, ultrasound and blood investigations like CBC, PT/INR of patient was monitored. No significant complications, including any form of hemorrhage, occurred and treatment with urokinase caused no significant changes in hematocrit, prothrombin time, partial thromboplastin time, and platelet countable. 
Gradually 1 week after urokinase instillation patient symptoms resolved and the collection reduced to $10 \mathrm{cc}$ in 2 week period after which the catheter was removed.

CT scan was performed at 1 month after drainage showed complete obliteration of abscess cavity.

Patient followed up monthly till 3 months, no recurrence seen.

Follow-up scans after urokinase instillation shows reduction in size of cavity with high density catheter in place and the cavity became smaller and eventually disappeared.

\section{DISCUSSION}

Percutaneous catheter drainage of abscesses is a welcome advance in the treatment of a disease in which mortality was high. Its benefits include the avoidance of general anesthesia, laparotomy, and prolonged postoperative hospitalization; cure rates, and those for morbidity and mortality, failure, and recurrences compare favorably with traditional surgery and the success rate varies between $76 \%$ and $89 \% .^{1,2,3}$ Failure is frequently due to multiloculation and phlegmon, fistula-associated abscesses, highly viscous content of the cavity, and improper catheter positioning. ${ }^{1}$ Complications include sepsis, contamination of the peritoneum, and empyema formation, and these occur at a rate of between $1.7 \%$ and $7.3 \%$.

Percutaneous needle aspiration combined with antibiotic therapy was safe, free from complications and effective. Its advantages over catheter drainage were that the patient suffered less discomfort, patient care was simpler, and costs were lower.

The most plausible explanation for the varied results with a septated abscess is that fibrin septa in such cavities may be complete or incomplete; when they are complete, drainage is poor, and when they communicate and are incomplete, curative drainage can occur.

Fibrin has long been regarded as one of the primary host defense mechanisms in intra-abdominal infections4. Leucocytes, which aggregate near a site of infection, release permeability factors such as histamine. These factors, in turn, lead to the exudation of fibrinogen and other proteins from surrounding blood vessels. Fibrinogen is converted to fibrin by tissue thromboplastin and helps to localize the spread of infection. ${ }^{5}$ Under physiologic conditions, fibrin is lysed by plasmin, a protease derived from the enzyme precursor plasminogen. Studies have shown that fibrinolytic activity depends on plasminogen activators, such as urokinase, streptokinase, and tissue plasminogen activator. ${ }^{5}$ Ongoing infection probably stimulates the conversion of fibrinogen to fibrin. Urokinase con- verts plasminogen to plasmin, which degrades fibrin and causes an increase in the fibrin split products.

Urokinase is derived from human kidney cell culture (Abbokinase, Abbott Laboratories, North Chi- cago, IL), was supplied as a sterile, lyophihized preparation. It was reconstituted with sterile water for injection, to a final dilution of $5000 \mathrm{lU} / \mathrm{mI}$.

Its inclusion criterias are quite broad; evidence of septa, loculi, hematoma, thick purulent material, or fluid with high CT attenuation values.

Exclusion criterias are age less than 18 or more than 95 years, CNS disorders (e.g., tumor, vascular problems), coagulation impairment including partial thrombophastin time more than $4 \mathrm{sec}$ beyond normal, platelet count less than 50,000, or low fibrinogen levels, hepatic failure, pregnancy, and

Abscesses in the spleen, pan- creas, or interloop area. Its Monitoring includes sequential CT scans; serial serum determinations of hematocnit, prothrombin time, partial thromboplastin time, platelet count, fibninogen levels, and levels of fibrin degradation products; and serial laboratory analysis of purulent material for fibrinogen and fibrin degradation products

The rationale for using urokinase to improve the results of percutaneous drainage of abscesses and fluid collections, and its effectiveness and safety, has recently been documented in the literature Park et al, showed that in vitro, the urokinase as compared with saline produced a considerable reduction in the viscosity of purulent material and its flow time through catheters of various sizes, and that the greatest benefit was achieved using smaller sizes of catheters. In addition, they stated that an extra theoretical benefit might be the lysis of fibrinous adhesions and loculations, which can also delay or impair drainage. ${ }^{6-10}$ The safety of urokinase in vivo was proved by Lahorra et al, who demonstrated that intracavitary urokinase produced an increased turnover of fibrin without any associated bleeding complication or changes in coagulation parameters. ${ }^{10}$ They stated, in addition, that urokinase also appeared to improve drainage of thick purulent material and to break any septations; in the above-mentioned study, all abscesses with loculations were successfully drained, as well as three infected hematomas in fungal or recurrent abscesses. In our case, CT scanning revealed a multiloculated abscess with a diameter of $8 \mathrm{~cm}$. According to the results of Lahorra et al, a total of 20,000 IU of urokinase solution was injected through the catheter into the abscess cavity during each instillation.

In this case, intracavitary urokinase instillation successfully enhanced drainage and broke any septations. On the basis of this initial experience, we suggest that if drainage after catheter placement is inadequate, urokinase should be used. 
Funding: No funding sources

Conflict of interest: None declared

Ethical approval: Not required

\section{REFERENCES}

1. van Sonnenberg E, Mueller PR, Ferrucci JT. Percutaneous drainage of 250 abdominal abscesses and fluid collections. Part 1: results, failures and complications. Radiology. 1984;151:337-41.

2. Gerzof SG, Johnson WC, Robbins AH, Nabseth DC. Expanded criteria for percutaneous abscess drainage. Arch Surg. 1985;120:227-32.

3. Johnson RD, Mueller PR, Ferrucci JT Jr. Percutaneous drainage of pyogenic liver abscess. AJR. 1985;144:463-7.

4. Ellis H, Harrison W, Hugh TB. The healing of peritoneum under normal and pathologic conditions. Br J Surg. 1965;52:471-6.

5. Dunn DL, Simmons AL. Fibrin in peritonitis: Ill. The mechanism of bacterial trapping in polymerizing fibrin. Surgery. 1982:92:513-9.

6. Lee KS, $1 \mathrm{~m}$ JG, Kim YH, Hwang SH, Bae WK, Lee $\mathrm{BH}$. Treatment of thoracic multiloculated empyema with intracavitary urokinase: a prospective study. Radiology. 1991;179:771-5.

7. Volgelzang RL, Tobin RS, Burstein S, Anschuetz SL, Marzan M, Kozlowski M. Transcatheter intracavitary fibrinolysis of infected extravascular hematoma. AJR. 1987;148:378-80.

8. Oh JH, Yoon Y. Case report of treatment of multiloculated liver abscess administration of urokinase through drainage catheter Journal of the Korean Radiological Society. 1995;32:479-81.

9. Park JK. Kraus FC, Haaga JR. Fluid flow during percutaneous drainage procedures: an in vitro study of the effects of fluid viscosity, catheter size, and adjunctive urokinase. AJR. 1993;160:165-9.

10. Lohora JM, Haaga JR, Stellato T, Flanigan T, Graham R. Safety of intracavitary urokinase with percutaneous abscess drainage. AJR. 1993;160:1714.

Cite this article as: Khade SA, Jadhav B, Meena P. Adjuvant Urokinase: percutaneous drainage of post lower segment cesarean section intra-abdominal abscess. Int J Reprod Contracept Obstet Gynecol 2019;8:2125-8. 